\title{
El sendero hacia la vacuna contra el SARS-CoV-2
}

\author{
DOI: https://doi.org/10.5377/alerta.v3i2.9749
}

\author{
José Eduardo Oliva Marín* \\ Departamento de Gobernanza y Gestión del Conocimiento del Instituto Nacional de Salud, San Salvador, El Salvador.
}

${ }^{*}$ Correspondencia

$\square$ joseduardoliva67@gmail.com

(1) 0000-0002-6005-0558

\section{Resumen}

El desarrollo de vacunas para infecciones virales se ha caracterizado por ser un proceso empírico y prolongado, basado en el uso de virus completos, atenuados o inactivados. La pandemia de COVID-19 ha activado el desarrollo de vacunas contra SARS-CoV-2. Una vez que la secuencia del genoma del virus estuvo disponible a mediados de enero 2020, diferentes laboratorios iniciaron el camino en busca de una vacuna. Las tecnologías que se están utilizando en la investigación de vacunas contra SARS-CoV-2 son: vacunas de ácido nucleico, vacunas proteicas recombinantes, vacunas virales basadas en vectores, vacunas vivas atenuadas y vacunas inactivadas. Doce vacunas están en etapa de ensayo clínico y 128 más en desarrollo preclínico. Aunque la elaboración de una vacuna viral toma un promedio de cinco a diez años, durante la crisis actual múltiples actores de varias naciones se han coordinado para que una vacuna contra SARS-CoV-2 esté lista en el menor tiempo posible, incluso antes de un año. Las vacunas AZD1222 de la Universidad de Oxford/AstraZeneca, Ad5-nCoV de CanSino Biologicals/Instituto de Biotecnología de Beijing y ARNm-1273 de ModernaTX Inc./Instituto Nacional de Alergia y Enfermedades Infecciosas de los Estados Unidos, son las candidatas más prometedoras.

\section{Palabras clave}

Ácidos nucleicos, proteínas recombinantes, vacunas atenuadas, vacunas de productos inactivados.

\section{Abstract}

The development of vaccines for viral infections has been characterized as an empirical and prolonged process, based on the use of complete, attenuated or inactivated viruses. The COVID-19 pandemic has activated the development of vaccines against SARS-CoV-2. Once the virus genome sequence was available in mid-January 2020, different laboratories began the journey in search of a vaccine. SARS-COV-2 vaccine research worldwide is testing the following technologies: nucleic acid vaccines, recombinant protein vaccines, viral vector-based vaccines, live attenuated vaccines, and inactivated vaccines. Twelve vaccines are in clinical trials, and 128 more, in preclinical stages. Although the development of a viral vaccine takes an average of five to ten years, during the current crisis, multiple actors from various nations have coordinated so that a SARS-CoV-2 vaccine is ready in the shortest possible time, even before one year has elapsed. AZD1222 vaccine from Oxford University/AstraZeneca, Ad5-nCoV from CanSino Biologicals/Beijing Institute of Biotechnology, and mRNA-1273 from ModernaTX Inc./United States National Institute of Allergy and Infectious Diseases, are the most promising candidates.

\section{Keywords}

nucleic acids, recombinant proteins, vaccines, attenuated, vaccines, inactivated
A ACCESO ABIERTO

SARS-CoV-2: the road in search of a vaccine

\section{Citación recomendada:}

Oliva Marín JE. El sendero hacia la vacuna contra el SARS-CoV-2. Alerta 2020;3(2):101-107. DOI: https://doi.org/10.5377/ alerta.v3i2.9749

\section{Recibido:}

23 de mayo de 2020

\section{Aceptado:}

30 de junio de 2020

\section{Publicado:}

8 de julio de 2020

\section{Contribución de autoría:} JEOM': elaboración del manuscrito y rendición de cuentas del mismo

\section{Conflicto de intereses:}

El autor declara no tener conflicto de intereses

\section{Introducción}

El desarrollo de vacunas requiere de tres etapas: una etapa de exploración, durante la cual se identifican antígenos naturales o sin- téticos; una etapa preclínica, que se lleva a cabo en cultivos celulares y en modelos animales; y una etapa de varias fases de ensayos clínicos en humanos, durante los cuales se confirma la seguridad, reactogenicidad e inmunogenicidad de la vacuna. Una vez 
elaborada, la vacuna debe registrarse en las agencias reguladoras correspondientes. En total, el proceso antes descrito toma de diez a quince años. Afortunadamente, durante la última década, han habido avances tecnológicos sustanciales que agilizan la concepción, el desarrollo, la fabricación y la entrega de una vacuna'.

La pandemia de COVID-19 ha activado el desarrollo de vacunas contra SARS-CoV-2. Una vez que la secuencia del genoma del virus estuvo disponible a mediados de enero de 2020, diferentes laboratorios iniciaron el camino en busca de una vacuna². El 16 de marzo de 2020, el Instituto Nacional de Alergia y Enfermedades Infecciosas de los Estados Unidos (NIAID, por sus siglas en inglés), inició el ensayo clínico Fase I (NCT04283461) de la vacuna ARNm-1273 desarrollada por ModernaTX Inc ${ }^{3}$. Dos días después, CanSino Biologicals/Instituto de Biotecnología de Beijing, China, reportaron el inicio del ensayo clínico Fase I (NCT04313127) de la vacuna Ad5-nCoV ${ }^{3}$.

El 3 de abril de 2020, líderes de los Institutos Nacionales de Salud de los Estados Unidos ( $\mathrm{NIH}$, por sus siglas en inglés), en coordinación con múltiples líderes de investigación y desarrollo de empresas biofarmacéuticas, junto con líderes de la Administración de Alimentos y Medicamentos de los Estados Unidos (FDA, por sus siglas en inglés), la Autoridad de Desarrollo e Investigación Biomédica Avanzada de los Estados Unidos, la Agencia Europea de Medicamentos (EMA, por sus siglas en inglés) y expertos académicos, buscaron identificar lagunas de conocimiento y/o tecnología y discutieron, además, oportunidades de colaboración para desarrollar una vacuna contra SARSCoV-24

Doce vacunas están en etapa de ensayo clínico y 128 más se encuentran en desarrollo preclínico ${ }^{3,5-10}$. Las vacunas candidatas abarcan diversas tecnologías que difieren en la eficacia con la que se estimula al sistema inmune, los mediadores inmunes movilizados, el volumen a aplicar, la vía de aplicación, la cantidad de refuerzos requeridos, la durabilidad de la protección y la frecuencia y/o gravedad de los efectos adversos ${ }^{11}$.

La precisión se logra al conocer la estructura atómica del antígeno contenido en la vacuna y al asegurar, que los epítopos virales a los que va dirigida, se conserven en la misma ${ }^{12}$. La mayoría de las vacunas candidatas inducirán anticuerpos neutralizantes específicos contra la proteína espiga $S$ de SARS-CoV-2, evitando así el acoplamiento del virus al receptor ECA2 humano ${ }^{13}$. La excepción a esta constante son las vacunas inactivadas, las cuales inducen la producción de anticuerpos contra diversas estructuras del microorganismo para el cual fueron desarrolladas.

\section{Desarrollo temático}

\section{Tipos de vacunas}

Las tecnologías que se están utilizando en la investigación de vacunas contra SARSCoV-2, son las vacunas de ácido nucleico (ADN o ARN), vacunas proteicas recombinantes, vacunas virales basadas en vectores, vacunas vivas atenuadas y vacunas inactivadas $^{13}$.

\section{Vacunas de ácido nucleico (ADN o ARN)}

En una vacuna clásica, un antígeno se introduce en el cuerpo para producir una respuesta inmune. Sin embargo, en el caso de las vacunas basadas en ADN o ARN, no se introduce un antígeno, se introducen fragmentos de ADN o ARN foráneos, conteniendo la información genética necesaria para que nuestro cuerpo sintetice uno o varios de los antígenos del microorganismo, contra el cual se desea generar inmunidad. Después de este paso, el mecanismo es similar al de las vacunas clásicas: el antígeno se presenta en la superficie de un subconjunto de células del sistema inmune, desencadenando la activación de las mismas ${ }^{14}$. Tras su absorción y expresión celular, los antígenos codificados en el ácido nucleico pueden provocar inmunidad humoral, así como respuestas inmunes mediadas por células ${ }^{15}$.

\section{Vacunas proteicas recombinantes}

Son vacunas producidas mediante tecnología de ADN recombinante. Esto implica insertar el ADN del microorganismo patógeno en células bacterianas o de mamíferos, para que estas expresen (repliquen) el antígeno contra el cual se desea obtener una respuesta inmune. Luego, este antígeno se purifica y se extrae para ser utilizado en la elaboración de las vacunas ${ }^{16}$. Los sistemas de expresión procariotas incluyen bacterias como la E. coli, así como células de mamíferos, levaduras o insectos. Los sistemas de expresión bacteriana se usan ampliamente debido a su facilidad de manejo y a su elevada capacidad de expresión ${ }^{17}$.

El ejemplo clásico de la vacuna proteica recombinante es la vacuna contra el virus de la hepatitis B (VHB). Se produce mediante la expresión del antígeno de superficie del VHB (HBsAg) utilizando un sistema de expresión de levaduras. Tras la expresión recombinante, el HBsAg se ensambla en partículas 
similares al virus, las cuales son extremadamente inmunogénicas, por lo que es una vacuna muy efectiva ${ }^{17}$.

\section{Vacunas virales basadas en vectores}

Esta tecnología emplea una variedad de virus vivos (replicantes, pero a menudo atenuados) o virus no replicantes, diseñándolos para que codifiquen los antígenos del patógeno contra el cual se necesita crear inmunidad. Estos antígenos son introducidos a las células del hospedero por el virus vector; una vez dentro, los antígenos se expresan y el hospedero es capaz de inducir respuestas inmunes contra el patógeno objetivo ${ }^{15,18}$. Los virus vivos replicantes tienen la ventaja de estimular tanto las células $B$, que producen anticuerpos, como las células T, que eliminan las células infectadas. Se han empleado diferentes tipos de virus para elaborar vacunas vectorizadas: adenovirus, lentivirus, parvovirus, togavirus, paramixovirus, rabdovirus y poxvirus ${ }^{15,19}$.

\section{Vacunas vivas atenuadas}

Las vacunas vivas atenuadas (VA) provienen de patógenos que se han atenuado mediante procesos de laboratorio. Se multiplicarán en el individuo vacunado, pero porque se han atenuado, no causarán ninguna enfermedad o esta será muy leve. Las WA estimulan una excelente respuesta inmune que es casi tan buena como la generada por el patógeno salvaje. Los microorganismos vivos proporcionan estimulación antigénica continua dando tiempo suficiente para la producción de células de memoria. En el caso de virus o microorganismos intracelulares, para los cuales generalmente se desea el desarrollo de inmunidad celular, los patógenos atenuados son capaces de replicarse dentro de las células hospedero. Las VVA producen inmunidad en la mayoría de los receptores con una sola dosis, excepto las administradas por vía oral. Como las WVA contienen organismos vivos, existe un grado de imprevisibilidad que genera preocupaciones sobre su seguridad y estabilidad. En casos esporádicos los patógenos atenuados mutan y se comportan como el microorganismo salvaje, causando enfermedades en los individuos vacunados o en sus contactos ${ }^{20,21}$.

\section{Vacunas inactivadas}

Estas vacunas están elaboradas con microorganismos que han sido inactivados a través de procesos físicos o químicos. Los microorganismos inactivados (muertos) no pueden causar enfermedad. Las vacunas inactivadas pueden no siempre inducir una respuesta inmune y la respuesta puede que no sea de larga duración. Por lo que usualmente se requieren múltiples dosis para evocar una inmunidad suficiente y duradera ${ }^{20}$. En general, la primera dosis no produce protección o inmunidad, pero prepara al sistema inmune. Una respuesta inmune protectora se desarrolla después de la segunda o tercera dosis. En contraste con las vacunas vivas, la respuesta inmune a una vacuna inactivada es principalmente humoral, obteniendo poca o nula inmunidad celular ${ }^{21}$.

\section{Coadyuvantes en desarrollo para COVID-19}

Para algunas tecnologías, los coadyuvantes mejoran la inmunogenicidad de las vacunas y reducen las dosis necesarias, lo que permite una cobertura más amplia sin comprometer la protección. Hasta ahora, al menos 10 farmacéuticas han expresado planes de desarrollar vacunas con coadyuvantes contra SARS-CoV-2. Algunas de estas compañías, incluyendo GlaxoSmithKline, Seqirus y Dynavax, se han comprometido a manufacturar diversos coadyuvantes autorizados (AS03, MF59 y CpG 1018, respectivamente) y hacerlos accesibles, para que sean utilizados en vacunas contra SARS-CoV-2 desarrolladas por otros laboratorios ${ }^{13}$.

\section{Situación actual}

Para el 16 de junio de 2020, el panorama global de vacunas contra COVID-19 incluye 140 vacunas en investigación; de los 140 proyectos, 128 están en etapa exploratoria o preclínica y 12 en etapa clínica. Las doce vacunas que han iniciado ensayos clínicos son las siguientes: ARNm-1273 de ModernaTX Inc./NIAID; Ad5-nCoV de CanSino Biologicals/ Instituto de Biotecnología de Beijing, China; AZD1222 de la Universidad de Oxford/AstraZeneca, Inglaterra; INO-4800 de la Farmacéutica Inovio, Estados Unidos; vacuna (sin nombre) del Instituto de Productos Biológicos de Wuhan/Sinopharm, China; vacuna (sin nombre) del Instituto de Productos Biológicos de Beijing/Sinopharm, China; PiCoVacc de la Corporation Sinovac Biotech, China; 3 LNPARNm de la fusión BioNTech(Alemania) / Fosun Pharma(China) / Pfizer(Estados Unidos); NVX-CoV2373 de Novavax, Estados Unidos; vacuna (sin nombre) del Institute of Medical Biology/Chinese Academy of Medical Sciences, China, y LV-SMENP-DC y aAPC patógeno específica, ambas del Instituto Medico Genoinmune de Shenzhen, China ${ }^{3,5-10}$. Las características individuales de cada vacuna se resumen en la Tabla 1. 
Tabla 1. Resumen de vacunas candidatas anti-SARS-CoV-2 en etapa clínica

\begin{tabular}{|c|c|c|c|}
\hline Laboratorio o Institución & Nombre y tipo de vacuna & Caracteristicas de la vacuna & Fase e identificador \\
\hline $\begin{array}{l}\text { ModernaTX Inc. /National } \\
\text { Institute of Allergy and } \\
\text { Infectious Diseases }\end{array}$ & $\begin{array}{l}\text { ARNm-1273 } \\
\text { Vacuna de ARNm encapsulado en } \\
\text { nanopartículas lipídicas (LNP, por sus } \\
\text { siglas en inglés) }\end{array}$ & $\begin{array}{l}\text { Contiene ARNm encapsulados en LNP, } \\
\text { que codifican la proteína espiga S de SARS- } \\
\text { CoV-2 }\end{array}$ & $\begin{array}{l}\text { Fase I } \\
\text { (NCT04283461) }\end{array}$ \\
\hline $\begin{array}{l}\text { CanSino } \\
\text { Biologicals/Beijing Institute of } \\
\text { Biotechnology }\end{array}$ & $\begin{array}{l}\text { Ad5-nCoV } \\
\text { Vacuna con vector adenovirus tipo } 5 \text { no } \\
\text { replicante }\end{array}$ & $\begin{array}{l}\text { Contiene un vector adenovirus tipo 5, que } \\
\text { acarrea la proteína espiga S de SARS-CoV-2 }\end{array}$ & $\begin{array}{l}\text { Fase I } \\
\text { (NCT04313127) } \\
\text { Fase II } \\
\text { (NCT04341389) }\end{array}$ \\
\hline $\begin{array}{l}\text { Inovio } \\
\text { Pharmaceuticals }\end{array}$ & $\begin{array}{l}\text { INO-4800 } \\
\text { Vacuna de plásmido de ADN }\end{array}$ & $\begin{array}{l}\text { Contiene plásmido de ADN aplicado mediante } \\
\text { electroporación, que codifica la proteína } \\
\text { espiga S de SARS-CoV-2 }\end{array}$ & $\begin{array}{l}\text { Fase I } \\
\text { (NCT04336410) }\end{array}$ \\
\hline $\begin{array}{l}\text { Shenzhen } \\
\text { Geno-Immune } \\
\text { Medical Institute }\end{array}$ & $\begin{array}{l}\text { aAPC patógeno } \\
\text { específica } \\
\text { Vacuna de células presentadoras de } \\
\text { antígenos artificiales (aAPCs, por sus } \\
\text { siglas en inglés), acarreadas por un vector } \\
\text { lentiviral }\end{array}$ & $\begin{array}{l}\text { La vacuna se desarrolló } \\
\text { utilizando un eficiente sistema vector lentiviral } \\
\text { para expresar minigenes de SARS-CoV-2 y } \\
\text { genes inmunomoduladores, modificando las } \\
\text { células presentadoras de antígenos artificiales y } \\
\text { activando las células T }\end{array}$ & $\begin{array}{l}\text { Fase I } \\
\text { (NCT04299724) }\end{array}$ \\
\hline $\begin{array}{l}\text { Wuhan Institute of Biological } \\
\text { Products/ Sinopharm }\end{array}$ & $\begin{array}{l}\text { Sin nombre } \\
\text { Vacuna inactivada }\end{array}$ & $\begin{array}{l}\text { Vacuna inactivada elaborada con SARS-CoV-2 } \\
\text { cultivado en células Vero }\end{array}$ & $\begin{array}{l}\text { Fase I/II } \\
\text { (ChiCTR2000031809) }\end{array}$ \\
\hline $\begin{array}{l}\text { Beijing Institute of Biological } \\
\text { Products/ } \\
\text { Sinopharm }\end{array}$ & $\begin{array}{l}\text { Sin nombre } \\
\text { Vacuna inactivada }\end{array}$ & $\begin{array}{l}\text { Vacuna inactivada elaborada con SARS-CoV-2 } \\
\text { cultivado en células Vero }\end{array}$ & $\begin{array}{l}\text { Fase I/II } \\
\text { (ChiCTR2000032459) }\end{array}$ \\
\hline Sinovac Biotech Co. & $\begin{array}{l}\text { PiCoVacc } \\
\text { Vacuna inactivada }\end{array}$ & $\begin{array}{l}\text { Vacuna de coronavirus inactivado y purificado } \\
\text { (PiCoVacc, por sus siglas en inglés), elaborada } \\
\text { con SARS-CoV-2 cultivado en células Vero }\end{array}$ & $\begin{array}{l}\text { Fase I/II } \\
\text { (NCT04352608) }\end{array}$ \\
\hline $\begin{array}{l}\text { BioNTech/Fosun Pharma/ } \\
\text { Pfizer }\end{array}$ & $\begin{array}{l}3 \text { LNP-ARNm } \\
\text { Vacuna de ARNm encapsulado en LPN }\end{array}$ & $\begin{array}{l}\text { Contiene ARNm encapsulados en LNP, que } \\
\text { codifican la proteína espiga S de SARS-CoV-2 }\end{array}$ & $\begin{array}{l}\text { Fase I/II } \\
\text { (NCT04368728) }\end{array}$ \\
\hline Novavax & $\begin{array}{l}\text { NVX-CoV2373 } \\
\text { Vacuna recombinante elaborada a partir } \\
\text { de subunidades } \\
\text { proteícas de } \\
\text { longitud total }\end{array}$ & $\begin{array}{l}\text { Contiene la proteína espiga S de } \\
\text { de SARS-CoV-2 en } \\
\text { nanopartículas, } \\
\text { con el adyuvante } \\
\text { Matriz M }\end{array}$ & $\begin{array}{l}\text { Phase I/II } \\
\text { NCT04368988 }\end{array}$ \\
\hline
\end{tabular}

Fuentes: National Institutes of Health/U.S. National Library of Medicine. ClinicalTrials.gov. 2020. Disponible en: https://clinicaltrials.gov. WHO: Draft landscape of COVID-19 candidate vaccines - 16 June 2020. Disponible en: https://www.who.int/publications/m/item/draft-landscape-of-covid-19-candidate-vaccines. The Chinese clinical test registration center. Chinese Clinical Trial Registry. 2020. Disponible en: http://www.chictr.org.cn/abouten.aspx. Mullard A. COVID-19 vaccine development pipeline gears up. Lancet. 2020; 395: 1751-52. DOI: 10.1016/S0140-6736(20)31252-6. 


\section{Panorama a corto plazo}

El 23 de abril de 2020 inició el ensayo clínico Fase I/II (NCT04324606) de la vacuna Chimpanzee Adenovirus Oxford 1 novel Coronavirus 19 (ChAdOx1 n-CoV19, por sus siglas en inglés), de la Universidad de Oxford. Su nombre fue modificado posteriormente a AZD1222. El ensayo ha evaluado la seguridad e inmunogenicidad de la vacuna en más de 1000 voluntarios sanos de 18 a 55 años, en varios centros de estudio de Inglaterra. De ser positivos los datos de este ensayo, se procederá con la Fase III, a desarrollarse de forma multicéntrica e internacional por AstraZeneca22.

El 6 de mayo, la FDA aceptó la solicitud de ModernaTX Inc./NIAID para que la vacuna ARNm-1273 procediera al ensayo clínico Fase II. ModernaTX Inc./NIAID están finalizando el protocolo para el ensayo clínico Fase III, programado para julio de $2020^{23}$.

Desde el 21 de mayo de 2020 AstraZeneca cuenta con el financiamiento de la Autoridad de Desarrollo e Investigación Biomédica Avanzada de los Estados Unidos (BARDA, por sus siglas en inglés), para el desarrollo, producción y distribución de la vacuna AZD1222. El programa de desarrollo incluye el ensayo clínico de Fase III con 30000 participantes y un ensayo clínico pediátrico. La meta es iniciar la distribución mundial de la vacuna, de una sola dosis, a partir de septiembre de 2020. La farmacéutica reconoce que la vacuna puede no funcionar, pero se ha comprometido a avanzar con el ensayo clínico con rapidez y llegar lo más pronto posible a la etapa de manufactura, a pesar del riesgo 22 .

Zhu $C$ et al. ${ }^{24}$ reportaron los primeros resultados de un ensayo clínico en humanos el 22 de mayo de 2020: la vacuna antiSARS-CoV-2 vectorizada por un adenovirus tipo 5, de CanSino Biologicals/Instituto de Biotecnología de Beijing. Fue tolerable e inmunogénica en adultos sanos durante su ensayo clínico Fase I (NCT04313127). La Fase I de este ensayo clínico fue llevada a cabo en China, administrando tres diferentes concentraciones de antígeno a los sujetos de estudio. La respuesta específica rápida de las células T se detectó el día 14 pos vacunación y las respuestas humorales específicas contra SARS-CoV-2, alcanzaron su punto máximo el día 28 después de la vacunación. Aunque observaron que la dosis alta de la vacuna tendía a ser más inmunogénica que las dosis media y baja, esta se asoció con una mayor reactogenicidad. El ensayo clínico Fase II (NCT04341389), ya en curso, proporcionará más información sobre la seguridad e inmunogenicidad de esta vacuna.
El 26 de mayo de 2020 Merck dio a conocer que desarrollará dos vacunas vectorizadas replicantes contra COVID-19. La primera, una vacuna vectorizada por el virus vivo atenuado de la estomatitis vesicular, diseñada por la Iniciativa Internacional de Vacunas contra el SIDA (IAVI, por sus siglas en inglés), para transportar la proteína espiga $S$ de SARS-CoV-2. El esfuerzo será financiado por el gobierno de los Estados Unidos a través de BARDA. Para la segunda vacuna, Merck está adquiriendo a Themis, una empresa austríaca que posee la tecnología de la vacuna viva atenuada contra el sarampión, desarrollada en el Instituto Pasteur ${ }^{25}$. En marzo de 2020 Themis, el Instituto Pasteur y la Universidad de Pittsburgh, recibieron financiamiento de la Coalición para las Innovaciones en Preparación para Epidemias (CEPI, por sus siglas en inglés), de Noruega, para desarrollar una vacuna vectorizada contra COVID-1926. Merk continuará con el desarrollo de esta vacuna en conjunto con Themis, utilizando el virus vivo atenuado del sarampión, como vector de la proteína espiga S de SARS-CoV-225.

La vacuna de Novavax, NVX-CoV2373, se considera una de las principales candidatas en la carrera de vacunas COVID-19. En mayo, Novavax recibió la mayor donación hasta la fecha de CEPI, para desarrollar su vacuna. A principios de este mes, la compañía ganó un contrato clave con el Departamento de Defensa de los Estados Unidos para suministrar NVX-CoV2373 a los militares²7.

\section{Desafíos}

Incluso con las tecnologías descritas anteriormente, el desarrollo de una vacuna contra SARS-CoV-2 plantea desafíos. Primero, aunque la proteína espiga $S$ del virus es un inmunógeno prometedor para generar protección, perfeccionar el diseño del antígeno es crítico para garantizar una respuesta óptima de inmunidad. El debate continúa sobre el mejor enfoque: utilizar la estructura completa de proteína de espiga S, o solo su dominio de unión al receptor ${ }^{28}$. Segundo, el desarrollo de vacunas efectivas contra el síndrome respiratorio agudo grave (SARS, por sus siglas en inglés) y el síndrome respiratorio del oriente medio (MERS, por sus siglas en inglés), se entorpeció por la falta de modelos animales adecuados para probarlas. El modelo animal ideal es aquel que se infecta con facilidad al inocularle el patógeno a investigar, desarrollando la fisiopatología y curso clínico de la enfermedad tal como se desarrolla en los humanos. A pesar que se presentaron en los modelos animales respuestas inmunológicas contra SARS-CoV y MERS-CoV, la replicación viral y las mani- 
festaciones clínicas de las enfermedades fueron escasas ${ }^{29,30}$. Esto produjo limitaciones para evaluar, en los modelos animales, la protección conferida por las vacunas ${ }^{29}$. Tercero, aunque las características óptimas de protección contra COVID-19 puedan inferirse de la experiencia adquirida durante la búsqueda de vacunas contra SARS y MERS, estas aún no se han establecido ${ }^{29,31}$.

Una vez elaborada la vacuna, existe un desafío adicional: la distribución equitativa de la misma. La necesidad de vacunas contra COVID-19 es global, aunque esta necesidad se distribuya de manera diferenciada entre las poblaciones. Las vacunas deberán ser priorizadas para el recurso humano dedicado a la atención de la salud y personas con mayor riesgo de enfermedades graves o muerte. Los países de altos ingresos no deberán monopolizar el suministro mundial de vacunas contra COVID-1932. Este riesgo es real. Durante la pandemia de Influenza A H1N1 en 2009, los países desarrollados negociaron grandes pedidos anticipados de la vacuna, desplazando a los países en vías de desarrollo ${ }^{33}$.

\section{Conclusiones}

Las tecnologías que están siendo utilizadas para desarrollar vacunas contra SARS-CoV-2 son las vacunas de ácido nucleico (ADN o ARN), las vacunas proteícas recombinantes, las vacunas vectorizadas, las vacunas vivas atenuadas y las vacunas inactivadas. Todas tienen ventajas y desventajas y mientras se encuentren en investigación, es difícil predecir cuál será la más exitosa. Las vacunas no se manufacturarán a tiempo para auxiliarnos durante el primer golpe de la pandemia. Sin embargo, si estas tienen éxito en los ensayos clínicos que se desarrollan actualmente, serán de utilidad para los embates subsecuentes de COVID-19; o bien, si el virus continúa circulando de manera estacional, serán útiles en el contexto pos pandemia.

Aunque la elaboración de una vacuna viral toma un promedio de 5 a 10 años, en la crisis actual múltiples actores de varias naciones se han coordinado para que una vacuna contra SARS-CoV-2 esté lista en el menor tiempo posible. Lo que ha hecho la diferencia, aparte de la urgencia y la enorme necesidad de la vacuna en el ámbito de la salud publica global, es la participación simultánea de las agencias regulatorias de los Estados Unidos, Europa y Asia, así como de la industria farmacéutica internacional.

SARS-CoV-2 está aquí para quedarse, al menos hasta que se desarrollen vacunas seguras y eficaces. La cobertura de inmunización contra COVID-19 debe desplegarse en todo el mundo para limitar el aparecimiento de nuevos brotes y para permitirnos, incluso, entrever la posibilidad de la erradicación. Las vacunas AZD1222 de la Universidad de Oxford/AstraZeneca, Ad5-nCoV de CanSino Biologicals/Instituto de Biotecnología de Beijing y ARNm-1273 de ModernaTX Inc./ NIAID, son las candidatas más prometedoras.

\section{Referencias Bibliográficas}

1. Graham BS, Mascola JR, Fauci AS. Novel Vaccine Technologies: Essential Components of an Adequate Response to Emerging Viral Diseases. JAMA. 2018;319(14):1431. DOI: 10.1001/jama.2018.0345

2. Lane R. Sarah Gilbert: carving a path towards a COVID-19 vaccine. The Lancet. 2020;395(10232):1247. DOI: 10.1016/S01406736(20)30796-0

3. U.S. National Library of Medicine. ClinicalTrials.gov. National Institutes of Health. 2020. Fecha de consulta: 21 de mayo de 2020. Disponible en: https://clinicaltrials. gov/

4. Collins FS, Stoffels P. Accelerating COVID-19 Therapeutic Interventions and Vaccines (ACTIV): An Unprecedented Partnership for Unprecedented Times. JAMA. 2020. DOI:

10.1001/jama.2020.8920

5. Kim YC, Dema B, Reyes-Sandoval A. COVID-19 vaccines: breaking record times to first-in-human trials. NPJ Vaccines. 2020;5(1):34. DOI: 10.1038/s41541-0200188-3.

6. Callaway E. The race for coronavirus vaccines: a graphical guide. Nature. 2020; 580(7805):576-7. DOI: 10.1038/d41586-02001221-y

7. WHO. Draft landscape of COVID-19 candidate vaccines. 2020. Disponible en: https://www.who.int/who-documentsdetail/draft-landscape-of-covid-19candidate-vaccines

8. The Chinese clinical test registration center. Chinese Clinical Trial Registry. 2020. Disponible en: http://www. chictr.org.cn/historyversionpuben. aspx?regno=ChiCTR2000032459

9. van Doremalen N, Lambe T, Spencer A, Belij-Rammerstorfer S, Purushotham JN, Port JR, et al. ChAdOx1 nCoV-19 vaccination prevents SARS-CoV-2 pneumonia in rhesus macaques. Microbiology. 2020. DOI: $\underline{10.1101 / 2020.05 .13 .093195}$

10. Gao Q, Bao L, Mao H, Wang L, Xu K, Yang M, et al. Development of an inactivated vaccine candidate for SARS-CoV-2. Science. 2020. DOI: 10.1126/science.abc1932 
11. Zhang L. Effect of Vaccine Administration Modality on Immunogenicity and Efficacy. Expert Rev Vaccines. 2015;14(11):1509-1523. DOI:10.1586/14760584.2015.1081067

12. Graham BS. Rapid COVID-19 vaccine development. Science Commentary. 2020 DOI: $10.1126 /$ science.abb8923

13. Thanh Le T, Andreadakis Z, Kumar A, Gómez Román R, Tollefsen S, Saville M, et al. The COVID-19 vaccine development landscape. Nat Rev Drug Discov. 2020;19(5):305-6. DOI: 10.1038/d41573-020-00073-5

14. Hubaud A. RNA vaccines: a novel technology to prevent and treat disease. Science in the News/Harvard University. 2015. Disponible en: http://sitn.hms.harvard. edu/flash/2015/rna-vaccines-a-noveltechnology-to-prevent-and-treat-disease/

15. Rauch S, Jasny E, Schmidt KE, Petsch B. New Vaccine Technologies to Combat Outbreak Situations. Front Immunol. 2018;9:1963. DOI: 10.3389/fimmu.2018.01963

16. Nature. Recombinant vaccines. Nature.com. 2020. Disponible en: https://www.nature. com/subjects/recombinant-vaccine

17. GeneScript. Recombinant vaccines. GeneScript/Make research easy. 2019. Disponible en: https://www.genscript.com/ recombinant-vaccine.html

18. Bouard D, Alazard-Dany N, Cosset F-L. Viral vectors: from virology to transgene expression. Br J Pharmacol. 2009;157(2):15365. DOI: $10.1038 /$ bjp.2008.349

19. Ramezanpour B, Haan I, Osterhaus A, Claassen E. Vector-based genetically modified vaccines: Exploiting Jenner's legacy. Vaccine. 2016;34(50):6436-48. DOI: 10.1016/j.vaccine.2016.06.059

20. World Health Organization. Vaccine safety basics learning manual. 2013. Disponible en: https://www.who.int/vaccine safety/ initiative/tech support/Naccine-safety-Ecourse-manual.pdf?ua=1

21. Centers for Disease Control and Prevention Epidemiology and Prevention of VaccinePreventable Diseases. Hamborsky J, Kroger A, Wolfe S, eds. 13th ed. Washington D.C.: Public Health Foundation. 2015.

22. Kemp A. AstraZeneca advances response to global COVID-19 challenge as it receives first commitments for Oxford's potential new vaccine. AstraZeneca Global Site. 2020. Fecha de consulta: 21 de mayo de 2020. Disponible en: https://www.astrazeneca. com/media-centre/press-releases/2020/ astrazeneca-advances-response-to-globalcovid-19-challenge-as-it-receives-firstcommitments-for-oxfords-potential-newvaccine.html

23. ModernaTX Inc. Moderna Announces Positive Interim Phase 1 Data for its mRNA Vaccine (mRNA-1273) Against Novel
Coronavirus. 2020. Disponible en: https:// investors.modernatx.com/node/8986/pdf

24. Zhu F-C, Li Y-H, Guan X-H, Hou L-H, Wang W-J, Li J-X, et al. Safety, tolerability, and immunogenicity of a recombinant adenovirus type- 5 vectored COVID-19 vaccine: a dose-escalation, open-label, nonrandomised, first-in-human trial. The Lancet. 2020. DOI: $10.1016 /$ S0140-6736(20)31208-3

25. Cohen J. Merck, one of Big Pharma's biggest players, reveals its COVID-19 vaccine and therapy plans. Science. 2020. Fecha de consulta: 26 de mayo de 2020. Disponible en: https://www.sciencemag. org/news/2020/05/merck-one-big-pharmas-biggest-players-reveals-its-covid-19vaccine-and-therapy-plans

26. Coalition for Epidemic Preparedness Innovations. CEPI collaborates with the Institut Pasteur in a consortium to develop COVID-19 vaccine. 2020. Disponible en: https://www.themisbio.com/wp-content/ uploads/2020/03/CEPI Pasteur-consortiumto-develop-COVID-19-vaccine 19032020 FINAL.pdf

27. Speights K. Better Coronavirus Stock: Moderna or Novavax? The Motley Fool. 2020. Disponible en: https://www.fool.com/ investing/2020/06/14/better-coronavirusstock-moderna-or-novavax.aspx

28. Du L, Zhao G, He Y, Guo Y, Zheng B-J, Jiang $S$, et al. Receptor-binding domain of SARS-CoV spike protein induces long-term protective immunity in an animal model. Vaccine. 2007;25(15):2832-8. DOI: 10.1016/j. vaccine.2006.10.031

29. Padron-Regalado E. Vaccines for SARS-CoV-2: Lessons from Other Coronavirus Strains. Infect Dis Ther. 2020;1-20. DOI: $10.1007 /$ s40121-020-00300-x

30. Sutton TC, Subbarao K. Development of animal models against emerging coronaviruses: From SARS to MERS coronavirus. Virology. 2015;479:247-58. DOI: 10.1016/j.virol.2015.02.030

31. Prompetchara, E. Immune responses in COVID-19 and potential vaccines: Lessons learned from SARS and MERS epidemic. Asian Pac J Allergy Immunol. 2020. DOI: $10.12932 / A P-200220-0772$

32. Yamey G, Schäferhoff $M$, Hatchett R, Pate M, Zhao F, McDade KK. Ensuring global access to COVID-19 vaccines. The Lancet. 2020;395(10234):1405-6. DOI: $10.1016 /$ S0140-6736(20)30763-7

33. Fidler DP. Negotiating Equitable Access to Influenza Vaccines: Global Health Diplomacy and the Controversies Surrounding Avian Influenza H5N1 and Pandemic Influenza H1N1. PLoS Med. 2010;7(5):e1000247. DOI: 10.1371/journal.pmed.1000247 